\title{
Calibration of local volatility using the local and implied instantaneous variance
}

\author{
Gabriel Turinici \\ CEREMADE, Université Paris Dauphine, \\ Place du Marechal de Lattre de Tassigny, 75016 Paris, France \\ gabriel.turinici@dauphine.fr
}

Dec 11th, 2008

\begin{abstract}
We document the calibration of the local volatility in terms of local and implied instantaneous variances; we first explore the theoretical properties of the method for a particular class of volatilities. We confirm the theoretical results through a numerical procedure which uses a Gauss-Newton style approximation of the Hessian in the framework of a sequential quadratic programming (SQP) approach. The procedure performs well on benchmarks from the literature and on FOREX data.
\end{abstract}

Keywords: calibration, local volatility, implied volatility, Dupire formula, adjoint, instantaneous local variance, instantaneous implied variance, implied variance.

\section{Introduction}

Consider a security $S_{t}$ (e.g. a stock, a FOREX rate, etc.) that follows the stochastic differential equation (cf. [Musiela and Rutkowski, 2005, Hull, 2006]),

$$
\begin{aligned}
& d S_{t} / S_{t}=(r(t)-q(t)) d t+\sigma d W_{t} \\
& S_{\mid t=0}=S_{0} \text { (given) }
\end{aligned}
$$

with $r(t)$ the time dependent risk-free rate, $q(t)$ the continuous dividend rate, $W_{t}$ a Brownian motion and $\sigma$ the volatility. We work with the local volatility model i.e. $\sigma=\sigma(S, t)$ (cf [Rubinstein, 1994, Dupire, 1994, Derman and Kani, 1994] for more detailed description); from a given $\sigma(S, t)$ one can compute vanilla call options prices for any strike $K$ and maturity $T$, denoted $C(S, t ; K, T, \sigma)$; we focus in this paper on the calibration of $\sigma(S, t)$ from quoted prices $C\left(S_{0}, 0 ; K_{l}, T_{l}, \sigma_{0}\right)$, $l=1, \ldots, L$ ( $\sigma_{0}$ is the unknown local volatility implied by the market data).

Building on theoretical results that we prove in some particular cases and complemented by positive numerical experiments, we document in this paper a calibration method that is specifically designed to invert the mapping from the instantaneous local variance to the instantaneous implied variance. This choice is rather natural since option traders often only quote the implied volatility and not the price). 
We show that in some particular cases our choice of variables render the optimization strictly convex, that it converges to the correct local volatility when the quoted options are dense enough and that Tychonoff regularization provides a stable way to approach the expected solution; moreover the convexity does not only speed the convergence but also eliminates local minima and operates a coherent selection of the adequate local surface, as confirmed in numerical experiments.

The solution is searched in a parametric space; our method can be seen as a particular case of sequential quadratic programming method ( hereafter SQP; more precisely IQP as inequality constraints are enforced at each QP step) with a Gauss-Newton type approximation of the Hessian (cf. [Nocedal and Wright, 2006, Bonnans et al., 2006]).

This approach is motivated by our target application : markets that only quote few maturities and strikes: e.g. FOREX risk reversals (quoted in terms of the implied volatility).

The balance of the paper is as follows: we give a short overview of the relevant literature in Section 1.1. Then we introduce the notations in Section 1.2 and give the main mathematical results in Section 2. The numerical implementation is given in Section 3. The results and concluding remarks are in Section 4.

\section{$1.1 \quad$ Literature overview}

The calibration of the local volatility from quoted marked prices can use the Dupire formula [Dupire, 1994, Hull, 2006] which is a direct relationship between a continuum of option prices (with varying strikes and maturities) and the local volatility.

However, when only a few prices are known, computing the derivatives in the Dupire formula is not effective and other methods have to be used [Avellaneda et al., 1997, Bodurtha and Jermakyan, 1999].

Among those, Coleman, Li \& Verma [Coleman et al., 2001] introduced a parametric procedure that we refine in this contribution. Further, L. Jiang et al. established a mathematical grounding for formulating this problem as a control problem [Jiang et al., 2003]; we will retain in this paper the adjoint state technique that we adapt to take into account the constraints (see [Lagnado and Osher, 1997, 1998] for related endeavors). Our procedure combines the approaches above and is accelerated by the use of an approximation of the functional through the use of the adjoint (15).

In all cases cited here the authors invert the mapping from local volatility to option prices.

\subsection{Notations and calibration variables}

Dupire equation ( see [Dupire, 1994, Hull, 2006, Achdou and Pironneau, 2005]) allows to compute a continuum of option prices $C\left(S_{0}, 0 ; K, T, \sigma\right)$ for all strikes $K$ and maturities $T$; to each $C\left(S_{0}, 0 ; K, T, \sigma\right)$ corresponds a implied volatility $\sigma^{I}(K, T)$ ( see [Black and Scholes, 1973]).

We have thus defined a map $\mathcal{P}$ from the local volatility $\sigma$ to prices i.e. $\mathcal{P}(\sigma)(K, T)=C\left(S_{0}, 0 ; K, T, \sigma\right)$. We also introduce the map $\mathcal{V}$ from the square $v=\sigma^{2}$ of the local volatility, named hereafter instantaneous local variance, to the square of the implied volatility, named hereafter instantaneous implied 
variance (both as functions of $K$ and $T$ ) i.e., with our notations $\mathcal{V}(v)(K, T)$ is the square $w=\left(\sigma^{I}(K, T)\right)^{2}$ of the implied volatility of the option with price $C\left(S_{0}, 0 ; K, T, \sqrt{v}\right)$.

To make our notations easier we will suppose from now on that the problem has a solution $\sigma_{0}$ (unknown to us); we denote $v_{0}=\sigma_{0}^{2}$.

Calibration can be recast as an inverse problem; most often used are the formulations $\mathcal{P}(\sigma)=\mathcal{P}\left(\sigma_{0}\right)$ or $\sqrt{\mathcal{V}\left(\sigma^{2}\right)}=\sqrt{\mathcal{V}\left(\sigma_{0}^{2}\right)}$; in practice this problem is solved by optimization of some 'least squares' quality functional to which one may add some Tychonoff regularization terms (see [Achdou and Pironneau, 2005, Bonnans et al., 2006] and latter in this paper).

A very useful property of the optimization functional is the convexity. If the convexity is not universal, we would like to have it at least on some distinguished classes of local volatilities. To the best of our knowledge, no systematic studies exist to motivate the choice of minimization variables. In particular none of the mappings $\sigma \rightarrow \mathcal{P}$ or $\sigma \rightarrow \sqrt{\mathcal{V}\left(\sigma^{2}\right)}$ has been shown to be convex on any particular classes of functions. On the contrary, for $\sigma^{2}=v \rightarrow w=\mathcal{V}(v)$ we give below a result for the situation when the local surface is strike independent but has (arbitrary) time dependence.

\section{Mathematical properties : strike independent local volatility}

The dependence $v \rightarrow w$ (see previous section for notations) is not straightforward to analyze, we refer to [Berestycki et al., 2002, Gatheral, 2006] for equations relating the two. Several asymptotics are available (cf. [Berestycki et al., 2002]) : if $\lim _{S \rightarrow 0} v(S, t)=v_{-}(t)$ and $\lim _{S \rightarrow \infty} v(S, t)=v_{+}(t)$ then

$$
\begin{aligned}
& \lim _{S \rightarrow 0} \mathcal{V}(v)(S, t)=\frac{1}{t} \int_{0}^{t} v_{-}(s) d s, \\
& \lim _{S \rightarrow \infty} \mathcal{V}(v)(S, t)=\frac{1}{t} \int_{0}^{t} v_{+}(s) d s .
\end{aligned}
$$

These relations speak to the convenience to use the map $v \rightarrow w$ (which is linear asymptotically) instead of e.g. $\sigma \rightarrow C$.

When the local variance depends only on time $v=v(t)$ we can further understand the properties of the optimization process; accordingly we will suppose for the rest of this section that the volatility is strike independent.

Lemma 1 The mapping $v \in L^{2}(0, T) \rightarrow\left\|\mathcal{V}(v)-\mathcal{V}\left(v_{0}\right)\right\|^{2}$ is strictly convex. In particular equation $\mathcal{V}(v)=\mathcal{V}\left(v_{0}\right)$ has an unique solution $\left(v_{0}\right)$.

Proof When $\frac{\partial \sigma(S, t)}{\partial S}=0 \forall S, t$, we know (see [Gatheral, 2006]) that $\mathcal{V}(v)(t)=$ $\frac{1}{t} \int_{0}^{t} v(s) d s$ and $\mathcal{V}(v)(0)=v(0)$. Thus $\mathcal{V}$ is a linear mapping in these variables. All that remains to be proved is that $V$ is non degenerate. We will prove more, namely that $V$ is a strictly positive operator. From the above formula we have $v=(t w)^{\prime} ;$ then $\left\langle\mathcal{V}(v), v>_{L^{2}(0, T)}=\int_{0}^{T}(t w)^{\prime} w d t=\frac{T}{2} w^{2}(T)+\int_{0}^{T} w^{2} d t\right.$ which is strictly positive except when $w$ is identically null. As a consequence we have that $\mathcal{V}$ is a continuous map from $L^{2}(0, T)$ to itself. The existence and uniqueness of the solution is a direct consequence of the positivity of the map. 
The lemma above ensures that the solution to the calibration problem $\mathcal{V}(v)=$ $\mathcal{V}\left(v_{0}\right)$ is unique; however the inverse mapping is not stable i.e. if we modify slightly (in $\left.L^{2}\right) \mathcal{V}\left(v_{0}\right)$ the solution $v_{0}$ can change dramatically. In order to lift the ill-possedness of the problem it is classical to add a regularization term [Achdou and Pironneau, 2005] which in this variable reads $\left\|v^{\prime}\right\|_{L^{2}}^{2}$. We will prove latter (Thm. 2) that adding this term gives indeed a stable way to invert the mapping.

However stability with respect to small variations in the overall volatility surface is not enough; in practice data does not cover all possible maturities but only a discrete set of times $T_{l}, l=1, \ldots, L$; a desirable property of the calibration process is the stability with respect to the amount of data available. The following result suggests a procedure that converges in a stable way:

Theorem 1 1/ For any division $\mathcal{T}=\left\{T_{0}=0 \leq T_{1} \leq \ldots \leq T_{l} \leq \ldots \leq T_{L}=T\right\}$ the minimum

$$
\min \left\{\left\|v^{\prime}\right\|_{L^{2}(0, T)}^{2} \mid v \in H^{1} ; \mathcal{V}(v)\left(T_{l}\right)=\mathcal{V}\left(v_{0}\right)\left(T_{l}\right), l=1, \ldots, L\right\}
$$

is attained in an unique point $v^{\mathcal{T}}$

2/ Suppose $v_{0} \in H^{1}(0, T)$. Then as $\max _{l=0, \ldots, L-1}\left(T_{l+1}-T_{l}\right) \rightarrow 0$ the sequence $v^{\mathcal{T}}$ converges to $v_{0}$ in $H^{1-\eta}$ for any $\eta>0$.

Proof 1/ The problem (5) can also be written as

$$
\min \left\{\left\|v^{\prime}\right\|_{L^{2}(0, T)}^{2} \mid v \in H^{1} ; \int_{T_{l}}^{T_{l+1}} v(t) d t=\int_{T_{l}}^{T_{l+1}} v_{0}(t) d t, l=0, \ldots, L-1\right\}
$$

Obviously $\left\{v \in H^{1} ; \mathcal{V}(v)\left(T_{l}\right)=\mathcal{V}\left(v_{0}\right)\left(T_{l}\right)\right\}$ is convex and closed $L^{2}$ and $H^{1}$. By taking a minimizing sequence $v_{n}$ we obtain by classical arguments that $v_{n}^{\prime}$ is $L^{2}$ bounded which, together with the weak lower semi-continuity of the norm gives the existence of a minimizer. The uniqueness follows from the strict convexity of the norm on the convex space of constraints. Let us note that the minimizer satisfies the following Euler equation $-v^{\prime \prime}=c s t$ on any $\left[T_{l}, T_{l+1}\right]$; in addition it is also continuous (as element of $H^{1}([0, T])$ ) and satisfies the other constraints $\int_{T_{l}}^{T_{l+1}} v(t) d t=\int_{T_{l}}^{T_{l+1}} v_{0}(t) d t, l=0, \ldots, L-1$.

$2 /$ For the second part we need a more detailed description of the solution. Let us denote $W^{\mathcal{T}}(t)=t \mathcal{V}\left(v^{\mathcal{T}}\right)(t), W_{0}(t)=t \mathcal{V}\left(v_{0}\right)(t)$ and recall that $v^{\mathcal{T}}(t)=\left(W^{\mathcal{T}}\right)^{\prime}(t)$. The minimization problem (5) of which $v^{\mathcal{T}}$ is solution can be rephrased in terms of $W^{\mathcal{T}}$ :

$$
W^{\mathcal{T}}=\operatorname{argmin}\left\{\left\|W^{\prime \prime}\right\|_{L^{2}(0, T)}^{2} \mid W \in H^{2}([0, T]), W\left(T_{l}\right)=W_{0}\left(T_{l}\right), l=0, \ldots, L\right\}
$$

We know that the solution $W^{\mathcal{T}}$ is the natural cubic spline interpolant of $W_{0}$ at points $T_{0}, \ldots, T_{L}$. The conclusion follows from the approximation properties of the cubic splines (cf. de Boor [2001], Quarteroni et al. [2007]).

At this point we have a stable procedure involving each time only a finite number of implied variances. But we still do not know how to compute in a stable manner the solutions $v^{\mathcal{T}}$. The answer is given in the next result; for simplicity we will omit to mark explicitly the dependence in $\mathcal{T}$. 
Theorem 2 The problem $\min _{v \in H^{1}} J^{\epsilon}(v)$ with

$$
J^{\epsilon}(v)=\sum_{l=1}^{L}\left(\mathcal{V}(v)\left(T_{l}\right)-\mathcal{V}\left(v_{0}\right)\left(T_{l}\right)\right)^{2}+\epsilon\left\|v^{\prime}\right\|_{L^{2}}^{2}
$$

has an unique solution $v^{\epsilon}$. Moreover $v^{\epsilon}$ converges in $H^{1}$ to $v^{\mathcal{T}}$ as $\epsilon \rightarrow 0$.

Proof The existence of $v^{\epsilon}$ follows from arguments of convexity; we refer the reader to the proof of Thm.3 in [Berestycki et al., 2002] where they use a very similar functional $\mathcal{V}$ (albeit over whole $\mathbb{R}$ and with other variables). They also prove that $v^{\epsilon}$ converges to some limit $v_{l}$ with $\mathcal{V}\left(v_{l}\right)\left(T_{l}\right)=\mathcal{V}\left(v_{0}\right)\left(T_{l}\right), l=1, \ldots, L$. We will now identify this limit $v_{l}$. Let $v$ be such that $\mathcal{V}(v)\left(T_{l}\right)=\mathcal{V}\left(v_{0}\right)\left(T_{l}\right)$, $l=1, \ldots, L$; from $J^{\epsilon}\left(v^{\epsilon}\right) \leq J^{\epsilon}(v)$ one obtains in particular $\left\|\left(v^{\epsilon}\right)^{\prime}\right\| \leq\left\|v^{\prime}\right\|$. Thus $\lim \sup _{\epsilon \rightarrow 0}\left\|\left(v^{\epsilon}\right)^{\prime}\right\| \leq\left\|v^{\prime}\right\|$. Recalling the weak lower semi-continuity of the norm and passing to infimum over all $v$ with $\mathcal{V}\left(v_{l}\right)\left(T_{l}\right)=\mathcal{V}\left(v_{0}\right)\left(T_{l}\right)$ one obtains

$$
\left\|\left(v_{l}\right)^{\prime}\right\| \leq \liminf _{\epsilon \rightarrow 0}\left\|\left(v^{\epsilon}\right)^{\prime}\right\| \leq \limsup _{\epsilon \rightarrow 0}\left\|\left(v^{\epsilon}\right)^{\prime}\right\| \leq\left\|\left(v^{\mathcal{T}}\right)^{\prime}\right\| .
$$

which, together with definition of $v^{\mathcal{T}}$ gives $v_{l}=v^{\mathcal{T}}$ and the convergence will be in $H^{1}$.

We give corresponding results for the situation of a parametric optimization in Appendix A.

\section{$3 \quad$ Numerical implementation}

We consider now the general dependence $\sigma=\sigma(S, t)$ and look for $v$ in a linearly constrained subset $\mathcal{K}$ of a finite dimensional linear space described in Section 3.2.

The surface $v$ minimizes the functional

$$
J^{\epsilon}(v)=\epsilon\|\nabla v\|_{L^{2}}^{2}+\sum_{l=1}^{L}\left(\mathcal{V}(v)\left(K_{l}, T_{l}\right)-\mathcal{V}\left(v_{0}\right)\left(K_{l}, T_{l}\right)\right)^{2}
$$

where the implied variances $\mathcal{V}\left(v_{0}\right)\left(K_{l}, T_{l}\right)$ are either quoted directly by the market or computed by inverting the Black-Scholes formula at the quoted values $C\left(S_{0}, 0 ; K_{l}, T_{l}, \sigma_{0}\right)$.

To find $v \in \mathcal{K}$ we use a sequential quadratic programming approach (SQP, see [Nocedal and Wright, 2006] Chap. 18) which, since the constraints on $\mathcal{K}$ are linear, reduces to the resolution at each iteration $v^{k}$ of the QP subproblem

$$
\min _{p}\left\{J^{\epsilon}\left(v^{k}\right)+D_{v} J^{\epsilon}\left(v^{k}\right)(p)+\frac{1}{2} D_{v v} J^{\epsilon}\left(v^{k}\right)(p, p) \mid v^{k}+p \in \mathcal{K}\right\}
$$

Then one sets $v^{k+1}=v^{k}+p^{k}\left(p^{k}\right.$ is the solution of the QP).

The cost of solving each QP is small; the costly part is the computation of the differentials $D_{v} J^{\epsilon}\left(v^{k}\right)$ and $D_{v v} J^{\epsilon}\left(v^{k}\right)$ which are usually replaced by approximations. Our choice is the following (a Gauss-Newton type of approximation, see eqn.(24)): $D_{v} J^{\epsilon}\left(v^{k}\right)$ is exact; in $D_{v v} J^{\epsilon}\left(v^{k}\right)$ we neglect terms containing $D_{v v} \mathcal{V}\left(v^{k}\right)\left(K_{l}, T_{l}\right)(l=1, \ldots, L)$ because they are multiplied by coefficients that tend to zero near the solution. 


\subsection{Differential}

We explain here how to compute $D_{v} \mathcal{V}(v)\left(K_{l}, T_{l}\right)$. Recall that the price $C^{l}(S, t)=$ $C\left(S, t ; K_{l}, T_{l}, \sqrt{v}\right)$ of a European call on $S_{t}$ with maturity $T_{l}$ and strike $K_{l}$ satisfies the (Black-Scholes) equation [Hull, 2006] for all $S \geq 0$ and $t \in\left[0, T_{l}\right]$ :

$$
\begin{aligned}
& \partial_{t} C^{l}+(r-q) S \partial_{S} C^{l}+\frac{v S^{2}}{2} \partial_{S S} C^{l}-r C^{l}=0 \\
& C^{l}\left(S, T_{l}\right)=\left(S-K_{l}\right)_{+}
\end{aligned}
$$

Remark 1 For a stock $q(t)$ is the (known) dividend rate while for a FOREX spot $q(t)$ is the foreign interest rate (with $r(t)$ being the domestic rate).

The price at $t=0$ of the contract is $C^{l}\left(S_{0}, 0\right)$. Using adjoint state techniques [Achdou and Pironneau, 2005, Jiang et al., 2003] the differential $D_{v} C^{l}\left(S_{0}, 0\right)$ is:

$$
D_{v} C^{l}\left(S_{0}, 0\right)=\frac{S^{2}}{2}\left(\partial_{S S} C^{l}\right) \chi
$$

Here the adjoint state $\chi$ (same for all options) is the solution of:

$$
\begin{aligned}
& \partial_{t} \chi+\partial_{S}((r-q) S \chi)-\partial_{S S}\left(\frac{v S^{2}}{2} \chi\right)+r \chi=0 \\
& \chi(S, t=0)=\delta_{S=S_{0}} .
\end{aligned}
$$

Both problems (12) and (15) can be solved e.g. through a Crank-Nicholson finite-difference scheme (in time and space) [Hull, 2006, Andersen and BrothertonRatcliffe, 1998] that we explain in Appendix B.

Remark 2 In the resolution of the adjoint state is is best to replace $\delta_{S=S_{0}}$ by the vector which is $1 / d S$ at $S_{0}$ and zero elsewhere, $d S$ being the spacial discretization step (this is the natural numerical discrete adjoint see [Conze et al., 2009] for an analysis).

To illustrate the nature of this gradient we display an example in Figure 1 where we note two singularities appearing in $\left(t=0, S=S_{0}\right)$ (from eqn (16)) and $\left(t=T_{l}=1, S=K_{l}\right)$ (from $\left.\partial_{S S}\left(S-K_{l}\right)_{+}\right)$(see also [Avellaneda et al., 1997] for similar conclusions).

Same technique works for any other quantity dependent on the price, e.g. the instantaneous implied variance $\mathcal{V}(v)\left(K_{l}, T_{l}\right)$. Recall that the explicit BlackScholes formula links the price and the implied volatility $C^{l}=C^{l}\left(\sigma^{I}\right)$ and as such $D_{v} \mathcal{V}(v)\left(K_{l}, T_{l}\right)=D_{v}\left(\sigma^{I}\right)^{2}=2 \sigma^{I} \frac{\partial \sigma^{I}}{\partial C^{l}} D_{v} C^{l}$. We recognize in the term $\frac{\partial C^{l}}{\partial \sigma^{I}}$ the Black-Scholes vega, that we will denote $\nu_{l, B S}^{I}$. We obtain

$$
D_{v} \mathcal{V}(v)\left(K_{l}, T_{l}\right)=2 \sqrt{\mathcal{V}(v)\left(K_{l}, T_{l}\right)} \frac{1}{\nu_{l, B S}^{I}} D_{v} C^{l}
$$

Note that for each term $\mathcal{V}(v)\left(K_{l}, T_{l}\right)$ of $J^{\epsilon}$ one needs to solve a PDE; the adjoint PDE is the same (i.e. $L+1$ PDEs in all). 


\subsection{Surface space and the construction of the QP}

A traditional choice to avoid singularities and address the possible non-uniqueness of the solution (for $\epsilon=0 J^{\epsilon}$ has an infinity of minima) is to parametrize the surface $\sigma(S, t)$ [Coleman et al., 2001]; the result will be the optimal surface in the class.

We give here a possible choice to describe the space of surface shapes. We consider a grid $S_{\min }=S^{0} \leq S^{1} \leq \ldots \leq S^{I}=S_{\max }$ (in $\left.S\right), t_{0}=0 \leq t_{1} \leq \ldots \leq$ $t_{J}=T=\max _{l} T_{l}$ (in $t$ ). We denote by $f_{i j}(S, t)$ the unique piecewise linear and continuous function that has value of 1 at $\left(S^{i}, t_{j}\right)$, and is zero everywhere else. The surfaces are linear combinations of the shapes $f_{i j}(S, t)$ :

$$
v(S, t)=\sum \alpha_{i j} f_{i j}(S, t) .
$$

The advantage of linear interpolation is that the shape functions have nice localization properties: the scalar product of two such functions (or their gradient) is zero except if they are neighbors i.e. matrices (21)-(22) are sparse. Also setting constraints e.g. $v(S, t) \in\left[v_{\min }, v_{\max }\right]$ for all $S, t$ is equivalent to asking that all $\alpha_{i j}$ are in $\left[v_{\min }, v_{\max }\right]$.

Remark 3 We also tested cubic splines interpolation and it performed equally satisfactory. Imposing the constraints $v(S, t) \in\left[v_{\min }, v_{\max }\right]$ in this case is done pointwise: we ask that $v\left(S^{i}, t_{j}\right) \in\left[v_{\min }, v_{\max }\right]$ in any interpolation node $\left(S^{j}, t_{j}\right)$; this reduces to a set of linear constraints in the coefficients $\alpha: v_{\min } \leq$ $\sum_{k l} \alpha_{k l} f_{k l}\left(S^{i}, t_{j}\right) \leq v_{\max }$.

We obtain a first order approximation formula around the current $v$ to be used in the QP subproblems in the same was as it is used in the nonlinear Gauss-Newton algorithm:

$$
\begin{aligned}
& \mathcal{V}\left(v+\sum_{i j} \alpha_{i j} f_{i j}(S, t)\right)\left(K_{l}, T_{l}\right)=\mathcal{V}(v)\left(K_{l}, T_{l}\right) \\
& +\sum_{i j} D_{v} \mathcal{V}(v)\left(K_{l}, T_{l}\right)\left(f_{i j}\right) \alpha_{i j}+o(\alpha) .
\end{aligned}
$$

Of course here $D_{v} \mathcal{V}(v)\left(K_{l}, T_{l}\right)\left(f_{i j}\right)=<D_{v} \mathcal{V}(v)\left(K_{l}, T_{l}\right), f_{i j}>_{L^{2}}$.

We denote

$$
Z_{l, a b}^{v}=D_{v} \mathcal{V}(v)\left(K_{l}, T_{l}\right)\left(f_{a b}\right) .
$$

Note that (19) already provides (some) second order information (i.e. we have already a part of the Hessian) for the second term of $J^{\epsilon}$ in eqn. (10).

For the Tychonoff regularization term $\epsilon\|\nabla v\|_{L^{2}}^{2}$ we will need the following matrices

$$
\begin{aligned}
& \left(Q_{S}\right)_{i j ; k l}=<\partial_{S} f_{i j}(S, t), \partial_{S} f_{k l}(S, t)>_{L^{2}} \\
& \left(Q_{t}\right)_{i j ; k l}=<\partial_{t} f_{i j}(S, t), \partial_{t} f_{k l}(S, t)>_{L^{2}} .
\end{aligned}
$$

A last ingredient involves bounds on $v(S, t)$; indeed, $v(S, t)$ cannot be negative. Even when this is the case, local volatilities with very low values (e.g. $3 \%$ !) are obviously not realistic. Enforcing constraints on the local volatilities is an important step towards selecting meaningful candidates. A choice that is 
consistent with the literature [Rubinstein, 1994, Derman and Kani, 1994] is to ask

$$
\begin{aligned}
v(t, S) & \in\left[v_{\text {min }}, v_{\text {max }}\right] \text { with } \\
v_{\text {min }} & =\left(\frac{1}{2} \min \left\{\sigma_{K_{l}, T_{l}}^{I ; \text { market }} ; l=1, \ldots, L\right\}\right)^{2}, \\
v_{\text {max }} & =\left(2 \max \left\{\sigma_{K_{l}, T_{l}}^{I ; \text { market }} ; l=1, \ldots, L\right\}\right)^{2} .
\end{aligned}
$$

\subsection{Optimization algorithm}

We interpolate available market data $\left(\sigma_{K_{l}, T_{l}}^{I ; \text { market }}\right)^{2}, l=1, \ldots, L$ to construct a surface $\mathcal{V}\left(v_{0}\right)$ to be fitted (the interpolation can be piecewise linear or cubic spline, etc). The initial guess $v^{0}$ is the projection of $\mathcal{V}\left(v_{0}\right)$ on the space $V e c t\left\{f_{i j}\right\}$; if the projection is not between bounds $v_{\min }$ and $v_{\max }$ we project again, pointwise, on bounds.

The SQP procedure operates by solving a quadratic programming (QP) problem around each point:

1/ For $v^{k}=\sum_{i j} \beta_{i j} f_{i j}(S, t):$ set $\left(B^{k}\right)_{l}=\mathcal{V}\left(v^{k}\right)\left(K_{l}, T_{l}\right)-\mathcal{V}\left(v_{0}\right)\left(K_{l}, T_{l}\right)$, compute $Z^{v^{k}}$ and solve the QP:

$\min _{\alpha}\left\{\left\|Z^{v^{k}} \alpha+B^{k}\right\|^{2}+\epsilon<\alpha+\beta,\left(Q_{S}+Q_{t}\right)(\alpha+\beta)>\mid v_{\text {min }}-\beta \leq \alpha \leq v_{\max }-\beta\right\}$.

$2 /$ is $\alpha^{\star}$ is the solution of the QP set $v^{k+1}=\sum_{i j}\left(\alpha_{i j}^{\star}+\beta_{i j}\right) f_{i j}(S, t)$; if the replication error is small then exit, otherwise set $k=k+1$ and return in $1 /$ for a new QP cycle.

In practice few QP cycles 1/-2/ are necessary: we tested on several indices and in the FOREX market and the numbers varied between 5 and 10 QPs.

Remark 4 In order to remain in a region where the approximation (19) holds we have also imposed constraints on the maximum change in $\alpha$ in step 2/. The bounds that proved satisfactory were of the order $\frac{v_{\max }-v_{\min }}{10}$ although we did not try to optimize these bounds.

Remark 5 The QP problem (24) can be solved by any suitable algorithm. The advantage of the approach is precisely to separate the optimization itself from the formulation of the problem. For instance Matlab uses by default a subspace trust-region method based on the interior-reflective Newton method described in [Coleman and Li, 1996].

We also tested a simple projected gradient: at each step we advanced a fixed step size in the direction of the gradient; then, points that do not satisfy the constraints $\left[v_{\min }, v_{\max }\right]$ are projected to either $v_{\min }$ or $v_{\max }$. We were surprised to see that in all cases the projected gradient performs as well as a general quadratic programming algorithm. We expect that the reason lies with the convenient choice of variables in which the problem was expressed i.e. $v=\sigma^{2}$ and $w=\mathcal{V}(v)$. This numerical observation may indicate further convexity of the functional $J^{\epsilon}(v)$ than was proved in Section 2. 


\section{Results and conclusions}

We used throughout (cf. eqn. (18)) a grid with $I=24$ equidistant values of $S$ centered in $S_{0}$; the discretization in $t$ used the grid $T_{0}=0, \frac{T_{0}+T_{1}}{2}, T_{1}, \ldots$, $\frac{T_{L-1}+T_{L}}{2}, T_{l}=T$. The regularization parameter $\epsilon$ was in the range [1.e $3,1 . e-2]$. The finite differences used 100 time steps and 200 spacial steps. The computations were performed with a Matlab/Octave code and took 2-3 minutes (wall-clock time for $10 \mathrm{QP}$ cycles) on a Intel(R) Xeon(TM) 3.20GHz CPU. For solving the QP both options were tested: the "quadprog" Matlab routine and the simple projected gradient described in Remark 5; both gave same results.

Remark 6 An alternative algorithm could be to fit directly the whole surface $\mathcal{V}(v)$ instead of an ensemble of fitting points $K_{l}, T_{l}$. The computation of the full surface $\mathcal{P}(\sigma)$ of option prices (then of $\mathcal{V}(v)$ ) can be performed directly from the Dupire equation at the price of one PDE (or from the equation relating local and implied volatilities cf [Berestycki et al., 2002, Gatheral, 2006]). But then we need a different adjoint PDE each time we want to compute the gradient for a new descent direction. The computational speed-up (or not) will depend on the relative number of gradient computations (for Dupire) and $L+1$ times the number of QP cycles (cf. Section 3.3) for the procedure we propose. As it will be seen below for our target applications (FOREX) we need between 5 and 10 QP cycles to converge and the data is not so abundant (30 known implied volatilities to fit).

An additional argument (but there are ways to circumvent this) is that we want to specifically focus on the points $K_{l}, T_{l}$ where we have market information and not fit the entire (necessarily interpolated) implied surface.

Let us now iterate through several benchmarks from the literature; we begin with the European call data on the S\& P index from [Andersen and BrothertonRatcliffe, 1998, Coleman et al., 2001] and use likewise in our computation only the options with maturities of less than two years. The initial index, interest rate and dividend rate are the same (see Figure 3 ). We first checked (not shown) that for $L=1$ the problem recovers the implied volatility; it did so with only one cycle. When we took all $L=70$ data the resulting local volatility surface is given Figure 3.

We next move to a FOREX example (from [Avellaneda et al., 1997]) where synchronous option prices (based on bid- ask volatilities and risk-reversals) are provided for the USD/DEM 20,25 and 50 delta risk-reversals quoted on August 23rd 1995. The results in Figure 4 show a very good fit quality with only five QP cycles $1 /-2 /$.

We remain in the FOREX market and take as the next example 10,25 and 50Delta risk-reversal and strangles for EUR/USD dated March 18th 2008 (courtesy of Reuters Financial Software). We recall that e.g. a 25 Delta risk reversal contract consists in a long position in a call option with delta $=0.25$ and a short position in a put option with delta $=-0.25$; the contract is quoted in terms of the difference of the implied volatilities of these two options. Note that the price of the options never appears in the quotes. In order to set the implied surface we used 10 and 25 Delta strangles which are quoted as the arithmetic mean of the implied volatilities of the two options above. Of course, from this data one can next recover the implied volatilities of each option, then all other 
characteristics. We give in Tables 1,2 and 3 the resulting data which is to be fitted; the data is to be read in the following way: for a given Delta and maturity, e.g. Delta $=0.25$ and $T_{l}=31$ days to expiry one finds its strike $K_{l}=1.6165$ in Table 1 , the implied volatility $\sigma^{I}\left(K_{l}, T_{l}\right)=12.95 \%$ from Table 2 and the premium (consistency check) $C\left(K_{l}, T_{l}\right)=0.0088 \%$ from Table 3 , all this with spot price $S_{0}=1.5755, r=r_{U S D}=0.2485$ and 'dividend' rate $q=0.0455$. We present in Figure 5 the implied and the calibrated local volatility. The procedure was also tested (not shown here) on other currencies pairs (GBP, CHF, JPY, KRW, THB, ZAR all with respect to USD as domestic currency) and performed well.

\section{Acknowledgements}

We gratefully thank two anonymous referees for their thoughtful feedback and relevant remarks that largely contributed to improve the paper; we also thank Mohammed Aissaoui, Marc Laillat and Kalide Brassier from the Adfin Analytics team of Thomson Reuters for providing the data in Tables 1,2 and 3 and for helpful discussions.

\section{Appendix A: parametric space versions of theo- retical results of Section 2}

In this section we would also like to know what happens when we look for the solution in a parametric space $\mathcal{K}$. A standard example is a bounded subset of a finite dimensional linear space. The requirement that at least one element exists that fits the data is a requirement on the diversity of elements of the set $\mathcal{K}$.

Theorem 3 Let $\mathcal{T}=T_{0}=0 \leq T_{1} \leq \ldots \leq T_{l} \leq \ldots \leq T_{L}=T$ be any division of $[0, T]$ and $\mathcal{K}$ be a compact closed subset of $H^{1}$ such that there exists at least one $v_{0, \mathcal{K}} \in \mathcal{K}$ with $\mathcal{V}\left(v_{0, \mathcal{K}}\right)\left(T_{l}\right)=\mathcal{V}\left(v_{0}\right)\left(T_{l}\right)$ for $l=1, \ldots, L$.

1/ The optimization problem

$$
\min \left\{\left\|v^{\prime}\right\|_{L^{2}(0, T)}^{2} \mid v \in \mathcal{K} ; \mathcal{V}(v)\left(T_{l}\right)=\mathcal{V}\left(v_{0}\right)\left(T_{l}\right), l=1, \ldots, L\right\}
$$

has an unique solution $v_{\mathcal{K}}$ which attains thus the minimum.

2/ For any $\epsilon>0$ the problem

$$
\min _{v \in \mathcal{K}} J^{\epsilon}(v)
$$

has an unique solution $v_{\epsilon, K} \in \mathcal{K}$; in addition the sequence $\left(v_{\epsilon, \mathcal{K}}\right)_{\epsilon>0}$ converges to $v_{\mathcal{K}}$ uniformly over $[0, T]$.

Proof $1 /$ The existence is straightforward due to the compactness of $\mathcal{K}$. The uniqueness uses the convexity of $\mathcal{K}$ and of the norm.

2 / Same arguments as the proof in the whole space apply (plus the compactness of $\mathcal{K}$ ). 


\section{Appendix B: the numerical discretization scheme}

We briefly explain in this section the resolution of equations (12) and (15). We use a standard finite differences scheme [Hull, 2006, Andersen and BrothertonRatcliffe, 1998]: denote by $C_{k}^{n}$ the approximation of the value $C\left(S_{k}, t_{n}\right)$ where $S_{k}=S_{\text {min }}+k \times d S$ is the $k$-th spatial point and $t^{n}=n \times d t$ the $n$-th time step; the equation for $C$ is backward, i.e. we know $C_{k}^{n+1}$ and want to compute $C_{k}^{n}$; the numerical scheme is then (we use $\theta=1 / 2$ ):

$$
\begin{aligned}
& \frac{C_{k}^{n}-\frac{C_{k+1}^{n+1}+C_{k-1}^{n+1}}{2}}{-d t}+(r-q) S_{k} \frac{C_{k+1}^{n}-C_{k-1}^{n}}{2 d S}-r C_{k}^{n} \\
& +\theta \frac{S_{k}^{2} v\left(S_{k}, t^{n}\right)}{2}\left\{\frac{C_{k+1}^{n}+C_{k-1}^{n}-2 C_{k}^{n}}{d S^{2}}\right\} \\
& +(1-\theta) \frac{S_{k}^{2} v\left(S_{k}, t^{n+1}\right)}{2}\left\{\frac{C_{k+1}^{n+1}+C_{k-1}^{n+1}-2 C_{k}^{n+1}}{d S^{2}}\right\}=0 .
\end{aligned}
$$

It is best to use for (15) the numerical adjoint of (12) (cf. also [Achdou and Pironneau, 2005] Section 8.3 for an example of numerical adjoint, albeit for the Dupire equation). This means that if the vector $C^{n}=\left(C_{k}^{n}\right)_{k \geq 1}$ solves the equation $\mathcal{A}^{n} C^{n}=\mathcal{D}^{n} C^{n+1}$ then the vector $\chi^{n}$ that approximates $\chi(\cdot, n \times d t)$ solves $\left(\mathcal{A}^{n}\right)^{T} \chi^{n}=\left(\mathcal{D}^{n-1}\right)^{T} \chi^{n-1}$. 


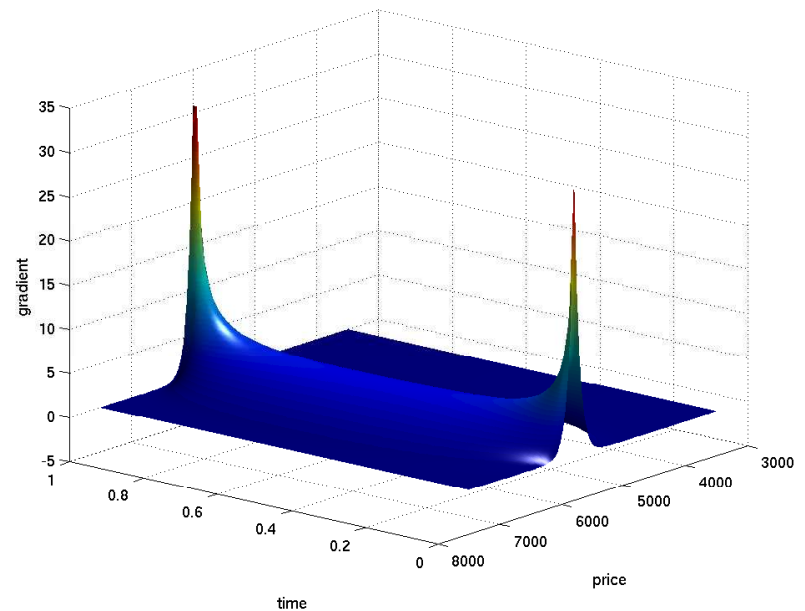

Figure 1: Differential $D_{v} C$ (see eqn. (14)) of the price $C$ of a plain vanilla call with respect to the local instantaneous variance $v$. Note the two singularities at the initial time (around the spot price) and at the expiration around the strike. These singularities prevent the direct use of a gradient method in these variables otherwise the resulting surface will be singular. 


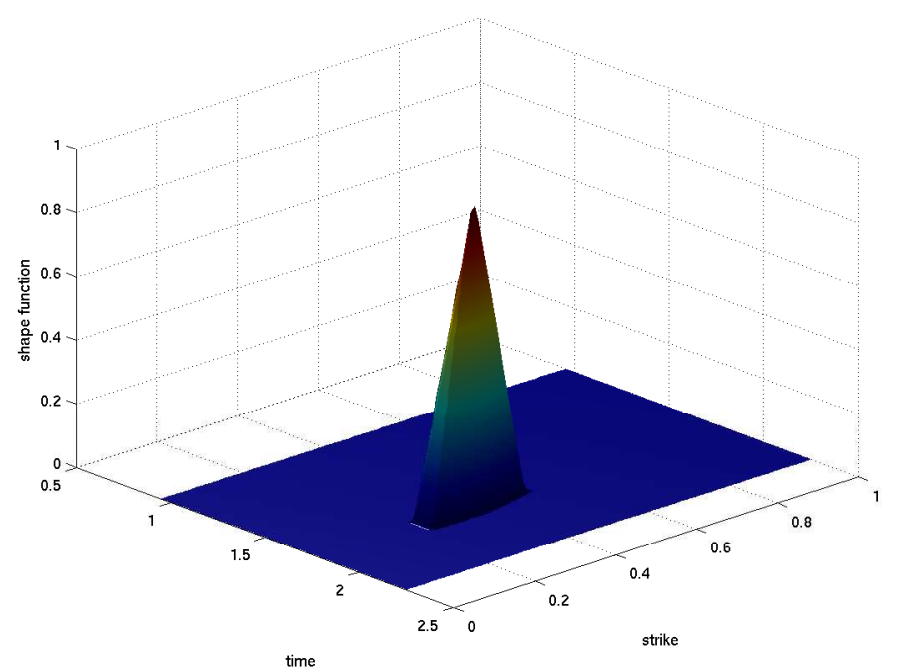

Figure 2: The local instantaneous variance $v$ is sought after as a linear combination of basic shapes $f_{i j}(S, t): v(S, t)=\sum_{i j} \alpha_{i j} f_{i j}$. A possible choice is to take $f_{i j}(S, t)$ as the (unique) linear interpolation which is zero except in some point $\left(S^{i}, t_{j}\right)$ (part of a grid in $S$ and $t$ ). We display here such a shape. 


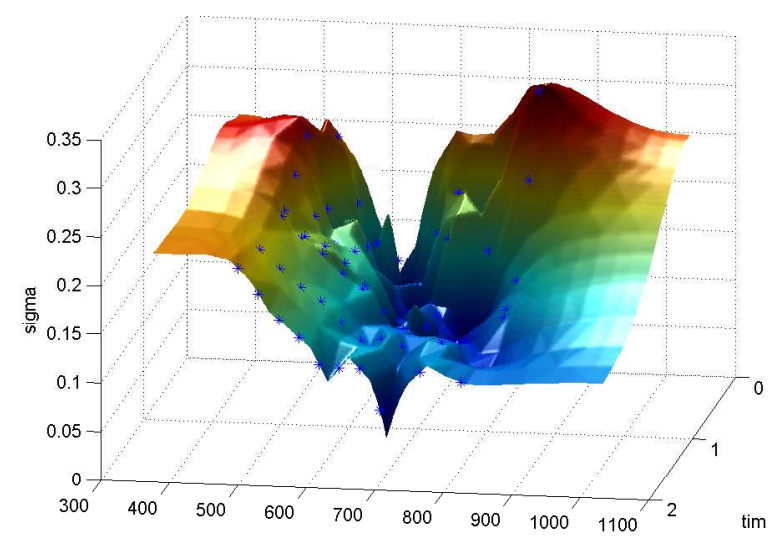

Figure 3: Local volatility surface of the S\&P 500 index as recovered from the published European call options data [Andersen and Brotherton-Ratcliffe, 1998, Coleman et al., 2001]; spot price is $\$ 590$; discount rate $r=6 \%$, dividend rate $2.62 \%$. The blue marks on the surface indicate the option prices that were used to invert i.e. the $K_{l}$ and $T_{l}(L=70)$. After $10 \mathrm{QP}$ cycles the prices are recovered up to $0.04 \%$ (relative to spot) and the implied volatility up to $0.28 \%$. Setting regularization parameter $\epsilon$ to smaller values gives better fit but less smooth surfaces. 

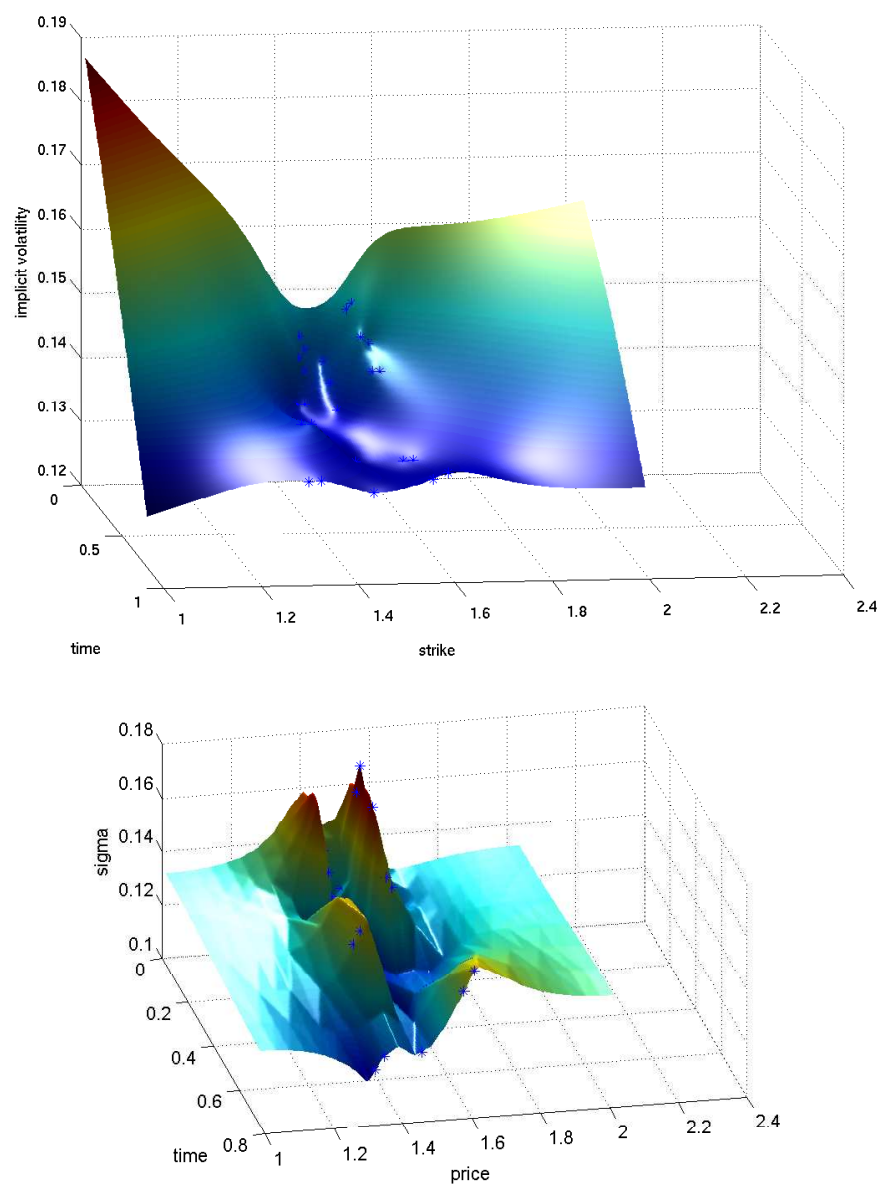

Figure 4: Top: implied volatility surface of the USD/DEM rate from [Avellaneda et al., 1997]; blue marks on the surface represent the available prices (to be matched). Bottom: local volatility surface as recovered from quoted 20,25 and 50-delta risk-reversals [Avellaneda et al., 1997]; (mid) spot price is 1.48875; $U S D$ discount rate $r=5.91 \%$, and DEM rate $4.27 \%$. The blue marks on the surface indicate the option prices that were used to invert i.e. the $K_{l}$ and $T_{l}$ $(L=30)$. After $5 \mathrm{QP}$ cycles the prices are recovered up to $0.005 \%$ (relative to spot), below the PDE resolution, and the implied volatility up to $0.03 \%$ (below the bid/ask spread). 


\begin{tabular}{|c|c|c|c|c|c|}
\hline Delta & 0,1 & 0,25 & 0,5 & 0,75 & 0,9 \\
\hline Days to Expiry & & & & & \\
\hline 7 & 1,6177 & 1,5965 & 1,5753 & 1,5544 & 1,5341 \\
31 & 1,6564 & 1,6150 & 1,5740 & 1,5335 & 1,4935 \\
59 & 1,6804 & 1,6253 & 1,5720 & 1,5191 & 1,4653 \\
92 & 1,7013 & 1,6333 & 1,5686 & 1,5042 & 1,4368 \\
184 & 1,7449 & 1,6474 & 1,5592 & 1,4711 & 1,3728 \\
365 & 1,8030 & 1,6611 & 1,5391 & 1,4164 & 1,2665 \\
\hline
\end{tabular}

Table 1: Strikes of the EUR/USD data derived from March 18th 2008 10,25 and 50 Delta risk-reversals and straddles used as input for the results in Figure 5.

\begin{tabular}{|c|c|c|c|c|c|}
\hline Delta & 0,1 & 0,25 & 0,5 & 0,75 & 0,9 \\
\hline Days to Expiry & & & & & \\
\hline 7 & $14,8250 \%$ & $14,1750 \%$ & $13,9250 \%$ & $14,1750 \%$ & $14,8250 \%$ \\
31 & $13,5250 \%$ & $12,9500 \%$ & $12,7750 \%$ & $13,1000 \%$ & $13,8250 \%$ \\
59 & $12,7750 \%$ & $12,1500 \%$ & $12,0250 \%$ & $12,4000 \%$ & $13,2750 \%$ \\
92 & $12,4250 \%$ & $11,7500 \%$ & $11,6250 \%$ & $12,1000 \%$ & $13,1250 \%$ \\
184 & $12,0875 \%$ & $11,2125 \%$ & $11,0500 \%$ & $11,6375 \%$ & $12,9625 \%$ \\
365 & $11,9125 \%$ & $10,8625 \%$ & $10,7000 \%$ & $11,3375 \%$ & $12,8875 \%$ \\
\hline
\end{tabular}

Table 2: Implied volatilities of the EUR/USD data derived from March 18th 2008 10,25 and 50 Delta risk-reversals and straddles used as input for the results in Figure 5.

\begin{tabular}{|c|c|c|c|c|c|}
\hline Delta & 0,1 & 0,25 & 0,5 & 0,75 & 0,9 \\
\hline Days to Expiry & & & & & \\
\hline 7 & 0,0015 & 0,0046 & 0,0121 & 0,0253 & 0,0425 \\
31 & 0,0029 & 0,0088 & 0,0231 & 0,0486 & 0,0824 \\
59 & 0,0038 & 0,0113 & 0,0298 & 0,0632 & 0,1088 \\
92 & 0,0046 & 0,0136 & 0,0358 & 0,0767 & 0,1341 \\
184 & 0,0063 & 0,0182 & 0,0479 & 0,1039 & 0,1883 \\
365 & 0,0086 & 0,0247 & 0,0650 & 0,1430 & 0,2724 \\
\hline
\end{tabular}

Table 3: Premiums of the EUR/USD data derived from March 18th 2008 10,25 and 50 Delta risk-reversals and straddles used as input for the results in Figure 5. 

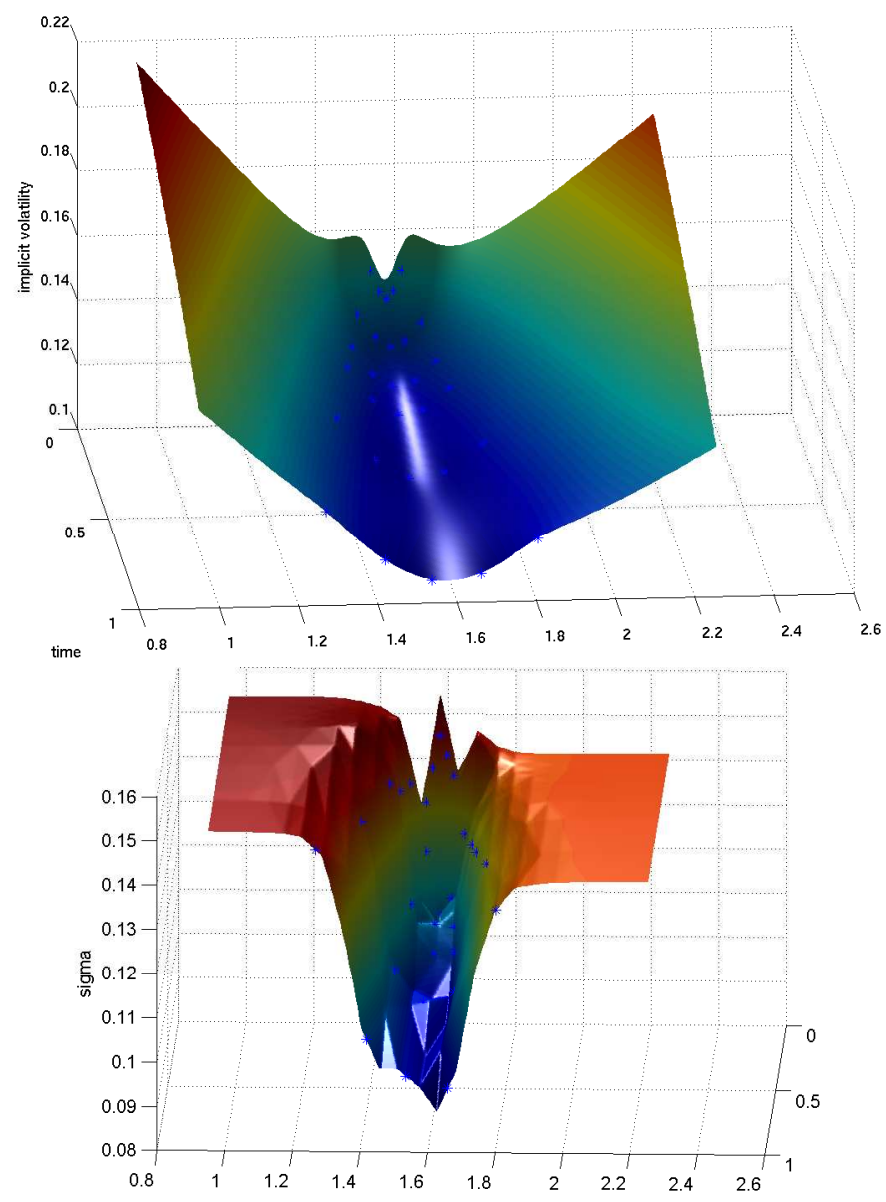

Figure 5: Top: implied volatility surface of the EUR/USD rate from Tables 1,2 and 3); marks on the surface represent the available prices (to be matched). Bottom: local volatility surface as recovered from quoted 10,25 and 50-delta risk-reversals and straddles; (mid) spot price is 1.5755 ; USD discount rate was set to $r_{U S D}=2.485 \%$, and $r_{E U R}=4.550 \%$. The blue marks on the surface indicate the option prices that were used to invert i.e. the $K_{l}$ and $T_{l}(L=30)$. After 5 QP cycles the prices are recovered up to $0.001 \%$ (relative to spot) and the implied volatility up to $0.02 \%$ (below the bid/ask spread). 


\section{References}

Yves Achdou and Olivier Pironneau. Computational methods for option pricing, volume 30 of Frontiers in Applied Mathematics. Society for Industrial and Applied Mathematics (SIAM), Philadelphia, PA, 2005.

L. Andersen and R. Brotherton-Ratcliffe. The equity option volatility smile: an implicit finite difference approach. The Journal of Computational Finance, 1: 5-32, 1998.

M. Avellaneda, C. Friedman, R. Holmes, and D. Samperi. Calibrating volatility surfaces via relative-entropy minimization. Applied Mathematical Finance, 4 (1):37-64, 1997.

H. Berestycki, J. Busca, and I. Florent. Asymptotics and calibration of local volatility models. Quantitative Finance, 2, 2002.

F. Black and M. Scholes. The Pricing of Options and Corporate Liabilities. Journal of Political Economy, 81, May-June 1973.

J. Jr. Bodurtha and M. Jermakyan. Non-parametric estimation of an implied volatility surface. J. Comput. Finance, 2:29-61, 1999.

J. Frédéric Bonnans, J. Charles Gilbert, Claude Lemaréchal, and Claudia A. Sagastizábal. Numerical optimization. Universitext. Springer-Verlag, Berlin, second edition, 2006. Theoretical and practical aspects.

Thomas F. Coleman and Yuying Li. A reflective Newton method for minimizing a quadratic function subject to bounds on some of the variables. SIAM J. Optim., 6(4):1040-1058, 1996.

Thomas F. Coleman, Yuying Li, and Arun Verma. Reconstructing the unknown local volatility function [J. Comput. Finance 2 (1999), no. 3, 77-100]. In Quantitative analysis in financial markets, pages 192-215. World Sci. Publ., River Edge, NJ, 2001.

A. Conze, N. Lantos, and O. Pironneau. The forward Kolmogorov equation for two dimensional options. Chinese Annals of Mathematics, to appear, 2008.

Carl de Boor. A Practical Guide to Splines. Applied mathematical sciences. Springer Verlag, Berlin, 2001.

E. Derman and I. Kani. Riding on a Smile. Risk, 7(2):32-9, February 1994.

B. Dupire. Pricing with a Smile. RISK, 7(1):18-20, 1994.

Jim Gatheral. The volatility surface : a practitioner's guide. John Wiley \& Sons, 2006 .

J. Hull. Options, Futures, and Other Derivatives. Prentice Hall, sixth edition, 2006.

Lishang Jiang, Qihong Chen, Lijun Wang, and Jin E. Zhang. A new well-posed algorithm to recover implied local volatility. Quantitative Finance, 3:451-457, 2003. 
R. Lagnado and S. Osher. Reconciling differences. Risk, 10(4):79-83, 1997.

R. Lagnado and S. Osher. A technique for calibrating derivative security pricing models: numerical solution of the inverse problem. J. Comput. Finance, 1: $13-25,1998$.

Marek Musiela and Marek Rutkowski. Martingale methods in financial modelling, volume 36 of Stochastic Modelling and Applied Probability. SpringerVerlag, Berlin, second edition, 2005.

Jorge Nocedal and Stephen J. Wright. Numerical optimization. Springer Series in Operations Research and Financial Engineering. Springer, New York, second edition, 2006.

Alfio Quarteroni, Riccardo Sacco, and Fausto Saleri. Numerical mathematics, volume 37 of Texts in Applied Mathematics. Springer-Verlag, Berlin, second edition, 2007.

M. Rubinstein. Implied binomial trees. J. Finance, 49:771-818, 1994. 